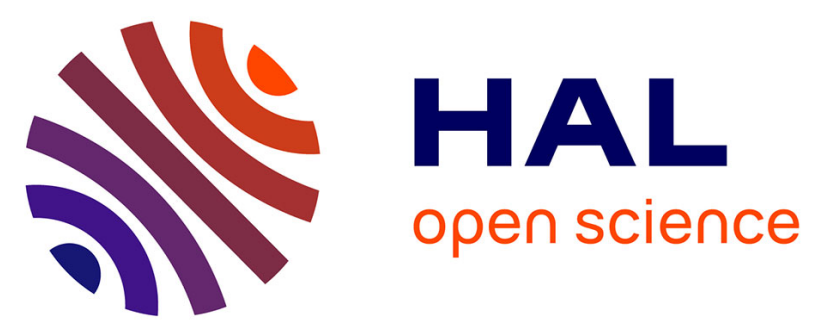

\title{
An operational perspective on potential uses and constraints of emerging tools for monitoring water quality
}

Nina Graveline, Laure Maton, Jean-Daniel Rinaudo, Helen Luckge, Eduard Interwies, Josselin Rouillard, Pierre Strosser, Kristine Palkaniete, Didier

Taverne

\section{To cite this version:}

Nina Graveline, Laure Maton, Jean-Daniel Rinaudo, Helen Luckge, Eduard Interwies, et al.. An operational perspective on potential uses and constraints of emerging tools for monitoring water quality. Trends in Analytical Chemistry, 2010, 29 (5), p. 378-384. 10.1016/j.trac.2010.02.006 . hal-00547408

\section{HAL Id: hal-00547408 https://hal-brgm.archives-ouvertes.fr/hal-00547408}

Submitted on 16 Dec 2010

HAL is a multi-disciplinary open access archive for the deposit and dissemination of scientific research documents, whether they are published or not. The documents may come from teaching and research institutions in France or abroad, or from public or private research centers.
L'archive ouverte pluridisciplinaire HAL, est destinée au dépôt et à la diffusion de documents scientifiques de niveau recherche, publiés ou non, émanant des établissements d'enseignement et de recherche français ou étrangers, des laboratoires publics ou privés. 


\title{
1 Potential uses and constraints of emerging water quality monitoring tools: an operational perspective
}

\author{
Nina Graveline ${ }^{1}$, Laure Maton ${ }^{1}$, Helen Lückge ${ }^{2}$, Josselin Rouillard ${ }^{3}$, Pierre Strosser ${ }^{3}$, \\ Kristine Palkaniete ${ }^{3}$, Jean-Daniel Rinaudo ${ }^{1}$, Didier Taverne ${ }^{4}$, Eduard Interwies ${ }^{2}$ \\ ${ }^{1}$ BRGM (French Geological Survey) Water department, 1039, rue de Pinville, 34000 \\ Montpellier, France. \\ ${ }^{2}$ InterSus - Sustainability Services - Chodowieckistr. 2 - 10405 Berlin, Germany \\ ${ }^{3}$ ACTeon, Le Chalimont, BP Ferme du Pré du Bois, 68370 Orbey, France \\ ${ }^{4}$ STS : centre de recherche Sciences, Territoires et Sociétés, Montpellier
}

Corresponding author Nina Graveline: email: n.graveline@brgm.fr; Tel : 00334671579 83, Fax : 0033467157975

\begin{abstract}
The European Water Framework Directive adopted in 2000 requires Member States to adapt and strengthen their monitoring of aquatic ecosystems. New monitoring strategies and practices have to be designed to monitor all polluting substances discharged into the aquatic environment, including priority substances or emerging pollutants that might be present at low concentration. This implies adapting monitoring locations and density, and monitoring frequency. It might also imply adapting monitoring techniques by integrating alternative Screening Methods and Emerging Tools for water quality monitoring to complement existing monitoring. The paper presents the results of five European case studies that explored the potential uses of Screening Methods and Emerging Tools for responding to the new monitoring challenges of the Water Framework Directive under different hydrological and environmental conditions. Combining their technical characteristics with practical needs identify by monitoring experts and water stakeholders, potential applications and opportunities for operational and investigative monitoring were identified. Advantages of these methods include the rapid delivery of results on site, their low cost or their capacity to acquire a larger number of observations within a given (short) time frame.
\end{abstract}

Keywords: WFD, water quality monitoring, biological techniques, early warning, screening methods, monitoring networks

\section{Introduction}

The implementation of the European Water Framework Directive (WFD 2000/60/EC [1]) will significantly change the monitoring of aquatic ecosystems in many European Member States. The WFD requires establishing monitoring strategies that combine: 1) surveillance monitoring to assess the risk of non compliance with WFD environmental objectives for all water bodies; 2) operational monitoring to assess the effectiveness of measures for improving water status/quality; and, 3) investigative monitoring for identifying unknown causes of contamination and supporting the identification of remediation actions. As a result, existing monitoring networks will have to be adapted to new requirements. In the majority of cases, the location and density of monitoring points will need to be adapted to provide an adequate spatial coverage (surveillance monitoring) and capture the effect of individual (main) pressures (operational and investigative monitoring). Furthermore, a larger group of 
substances have now to be monitored in a more systematic manner, in particular those listed as priority substances, as specified by council directive 2008/105/EC [2].

From a technical perspective, the main challenges will consist of establishing new monitoring networks (selection of representative monitoring points), developing information systems for managing an increasing volume of data coming from different producers [3], developing new analytical methods and controlling measurement uncertainty [4]. From an economic perspective, the challenge will be to minimize monitoring cost. In some cases, organisational changes might also be necessary, with possible redistribution of tasks and responsibilities within or between organisations, be it private, public, national and/or regional actors. This changing context may offer new opportunities for the development of techniques that differ from the traditional spot (bottle or grab) sampling and laboratory analysis. The Common Implementation Strategy (CIS) guidance document on surface water chemical monitoring [5] identifies several new monitoring methods which are referred to as Screening and Monitoring Emerging Tools (SMETs). This paper discusses the potential uses and constraints of these SMETs.

The term "Screening and Monitoring Emerging Tools" (SMETs) is used here to design tools that differ from classical spot sampling and laboratory analysis. They can be used directly on-site or in-situ, and they often enable a quicker water quality assessment than with classical lab analysis. Different types of SMETSs can measure time weighted average concentrations of pollutants, provide rapid on-site or on-line analysis or detect potentially harmful conditions through biological or chemical detectors. The term SMETs encompasses a large variety of technologies including: (i) equipment for measuring physico-chemical characteristics; (ii) biological assessment techniques (e.g. biomarkers, bioassays/biosensors and biological early warning systems); and, (iii) chemical analytical or sampling methods that can be used on- or in site (e.g. sensors, passive sampling devices, test kits, immunoassays). A detailed review of these tools can be found in Allan et al. [6] and Greenwood et al.[7].

The technical and economic potential offered by SMETs is likely to differ widely across Europe to reflect the heterogeneity of existing monitoring networks, organisation of actors (public / private), technology and labour cost structure, monitoring culture (engineering-driven or not). This paper investigates their potential uses from the perspective of water monitoring experts and stakeholders in charge of implementing the Water Framework Directive and its monitoring requirements. It describes both opportunities and constraints and give precious indication on the potential integration of these innovative tools in the WFD monitoring programs. The work is based on five European case studies conducted as part of the SWIFT-WFD EU research project in the Czech Republic, France, Germany, Latvia and the United Kingdom.

The reminder of this paper is organised as follows. The following section presents a typology of possible uses of SMETs. The third section presents how SMETs are perceived by monitoring experts and practitioners. The fourth section then focuses on constraints that may limit the use of SMETs. The paper concludes by discussing possible options for removing these constraints. 


\section{SMETs' technical characteristics from a user perspective}

98 Four main criteria can be used to classify SMETs from a user's perspective: Criteria 1: where the measurement is made - on site (including in situ measurements) or in a laboratory, after sampling; Criteria 2: type of sampling protocol (spot sampling, passive sampling, 24 hours average sample or no sampling (continuous measurement)); Criteria 3: type and accuracy of the measurement (binary response if the contaminant is present, quantification above a certain concentration) and its specificity (does it detect a single substance, a group of substance or a total toxic effect - for instance with biological early warning systems such as trout, that react according their sensitivity to a general water quality); Criteria 4: sensitivity towards contaminants (including detection limits).

Overall, there are different reasons why SMETs can change daily practices of monitoring:

(i) A large number of SMETs can be used on site and be deployed quickly (sensors, test kits), allowing the production of a large quantity of data in a short period of time. This is relevant to screening purposes (in space or in time), when the objective of the water quality survey is to detect the presence of a contaminant over a large area (or with a high time resolution), without necessarily quantifying its concentration.

(ii) Passive samplers or on-line sensors allow the monitoring of concentration over time (cumulated weighted average or high time resolution) (even if passive samplers are just a sampling tool and not a monitoring one as it needs further lab analysis); their use can help assessing the total load carried by a stream over a given period of time, an objective that is more difficult and expensive to obtain with spot sampling.

(iii) Passive samplers can concentrate the presence of traces of contaminant (e.g. pharmaceutical substances) which could not be detected with traditional sampling.

(iv) Some SMETs (e.g. Biological Early Warning Systems - BEWS- like fishes) can help assessing the overall toxicity of all contaminants, without identifying the specific substance(s) causing the problem. This might help water managers to rapidly detect problematic areas or time periods. Other BEWS can assess a modification of the medium reacting to a sudden change of $\mathrm{pH}$ or of temperature.

As illustrated above, SMETs are particularly useful when they provide a different type of information than obtained with traditional spot sampling and laboratory analysis.

\section{Approach and methodology}

As a result of their technical characteristics, SMETs can deliver several functions corresponding to some of the WFD monitoring requirements. Clearly, however, the decision to integrate SMETs into existing monitoring strategies will account for the demand and operational constraints faced by water monitoring experts.

To capture the demand and perception of water monitoring experts vis-à-vis SMETs, five river basins where selected throughout Europe for in-depth investigation. These include: the Ribble in England; the Daugava in Latvia; the Aller in Germany; the Orlice in Czech Republic; and, the French part of the Upper Rhine. Research activities were carried out in these basins to investigate the potential integration of SMETs into 
existing monitoring networks and campaigns. The case studies cover diverse environmental issues (agricultural, industrial and urban pollution) and water bodies (small and large rivers, aquifers, coastal and transboundary water bodies). In each of these basins, the demands, expectations and perceptions of potential users of SMETs were collated via individual interviews and collective meetings. For example, a total of 50 water monitoring experts or practitioners were met as part of the French Upper Rhine case study, representing staff from the environmental-water administration, communities in charge of local networks, health services (in charge of drinking water quality and monitoring), industries and private companies (in charge of monitoring or working with water quality data) and non governmental organisations.

The methodology carried out was based on four main steps.

The first step built on a series of "face to face" interviews, based on a questionnaire that dealt with: (i) the profile of the expert interviewed and of its organisation; (ii) the description and evaluation of existing monitoring networks and water quality data (on technical, organisational and economic aspects); (iii) expected future changes in existing monitoring networks (in particular to respond to the WFD new requirements); and (iv) the potential for SMETs application for water quality surveys. A presentation of SMETs and of their general technical characteristics was included in the interview using a simple leaflet presenting the characteristics of the main SMETs (on-line systems, passive samplers, portative lab-instruments, electrochemical sensors, probes, bioassays, immunoassays and biological early warning systems...).

Based on the analysis of the results of the interviews and of existing documents dealing with water quality monitoring (study reports, national regulation, monitoring guidelines etc.), the second step identified specific potential uses for SMETs in each case study. This helps highlighting problems faced today for the collection of monitoring data, the organisation of data, their analysis and interpretation and their dissemination. Problems identified by producers, managers or users of data in current water quality monitoring systems were compared with the strengths of SMETs that could help solving these.

In parallel, real field testing of SMETs was carried out in each case study area (see [8] and [9]). An economic evaluation of the new information SMETs would deliver was also carried out (see [10]). And further experts feedbacks were collected to understand people's perception and acceptance vis-à-vis SMETs.

The forth and final step aimed at presenting, sharing and debating results with a wider audience by organising European, national and basin scale workshops . This further helped refining the description of potential uses for SMETs in water quality monitoring and the identification of their main constraints and opportunities within the WFD implementation context. Reports on workshops results are available at internet website: www.swift-wfd.com.

\section{Potential uses of SMETs}

Although experts' consultation stressed today's limited demand for alternative monitoring techniques, public organisations concerned with environment or health agreed that most existing monitoring networks would need to be redesigned to improve knowledge on water quality. Water monitoring experts highlighted the roles SMETs could play for developing an effective monitoring network. 


\subsection{SMETs for designing monitoring networks}

Before establishing new monitoring networks, SMETs can help capturing the spatial and temporal variability of pollutant concentrations as pre-requisite to the selection of representative monitoring stations. SMETs can be particularly interesting when it is difficult to choose representative points such as in groundwater bodies. For example, sensors and passive samplers could be used to assess concentration temporal variability before optimising monitoring frequency. This application was suggested by stakeholders consulted in the UK, the Czech Republic and Germany.

\subsection{Surveillance monitoring in future risk assessment and WFD cycles}

When designing surveillance monitoring networks, choosing substances representing a significant environmental risk, including emerging pollutants, remains a clear challenge. "Emerging pollutants" are by definition not monitored today by current surveillance networks. Surveys of a few months could be done with selective passive sampling on integrative points (e.g. outlet of basins) to identify new pollutants to be monitored in the future. Passive sampling enables to catch very low pollutant concentrations. They can help detecting pollution at an "early stage", for example for pharmaceuticals and hormones discharged by wastewater treatment plants. The use of SMETs in surveillance monitoring was seen as particularly relevant for the Latvian case, where many water bodies lack monitoring. On the opposite, French, British and Czech experts saw limited potential for SMETs in surveillance monitoring in their countries. Other tools such as BEWS or online sensors could be used to assess long term trends. However, their use could be restricted by the problem of dataset continuity over long time series (historical data acquired with traditional techniques are not comparable with new data collected with SMETs, explaining why experts may prefer to stick to existing monitoring techniques).

\subsection{Operational monitoring}

Old industrial sites often represent a risk that is poorly characterised, in particular when pollution plumes are present in the soil or sub-soil. Monitoring the propagation of pollution plumes in these sites, which are common in many parts of Europe, is crucial to: (i) secure drinking water resources that might be threaten by pollution propagation; and, (ii) to evaluate the efficiency of remediation measures that are, or have been, implemented. The use of SMETs could increase the frequency of monitoring at the border, and downstream, of contaminated sites. Sensors in wells and passive samplers that could be installed and retrieved every month for instance, could be used. This opportunity has been emphasized by experts consulted in France and Latvia where many water bodies are significantly affected by industrial pollution for which operational monitoring is mandatory.

\subsection{Investigative monitoring}

In all five case studies, investigative monitoring is seen as having the greatest potential for SMET applications. Where a recurrent pollution can be observed within a natural network of rivers or a waste water collection network, specific field tools can be applied to identify potential point emission sources. Easy to use, SMETs could be applied on-site or in situ, for example sensors, biosensors, bioassays or immunoassays 
(pesticides), providing the possibility to carry out a large number of measures in a single. To search for PCBs within a wastewater network is an example where the application of SMETs would help identifying the source of PCBs that currently deteriorates the quality of sludge, making it improper for manure spreading on agricultural fields. Similar investigations could also be proposed to identify the origin of mercury pollution that is currently unknown (probably an old industry) in the Thur River in Alsace.

SMETs could help revealing the existence of rare and sudden pollution peaks that can not be detected by existing monitoring systems but that can be responsible for ecological disorders (e.g. fish mortality, bioaccumulation of pollutants in fish flesh). Biological Early Warning Systems (BEWS) or on-line systems could monitor continuously indicator parameters ( $\mathrm{pH}$, conductivity, TOC) or more specific parameters such as pesticides (Fluotox for instance). This would help linking pesticides pollution "peaks" to practices of potentially polluting activities (green spaces treatment in cities or agricultural practices) and/or to climatic events (rainfalls).

SMETs could also help detecting sources and plumes of pollution in very large industrial sites characterised by the presence of numerous pollution sources, substances and plumes. In such sites, high spatial resolution mapping could be carried out with SMETs to identify the principal sources of pollution. It could, then, help finetuning remediation measures.

\section{5. ..and beyond the WFD}

There are clear opportunities for using SMETs beyond the WFD, in some cases in areas where SMETs are already applied today. In particular:

(i) Alarm and continuous monitoring for strategic water resources such as a drinking water abstraction points can be proposed to reduce potential risks linked to the operation of large industrial sites or to urban discharges. Early detection of pollution would enable early remediation and prevention. Permanent Biological Early Warning Systems (BEWS) stations can be installed at the boarder between regions or countries, similar to the alarm station that already exists in Huningue (south of the Alsace region) at the Swiss-German-French border for protecting the (strategic) Alsace aquifer (used for drinking water) from accidental pollution. They are also used at the entry of wastewater treatment stations to protect their biological functioning from toxic discharges. Lastly, they can be used by industries with high water quality requirements.

(ii) Biological or very rapid tools can also confirm a "suspicion" in the case of an accident or of abnormal field observation data. In a regulatory context of discharge control, and when SMETs will be standardized, they could be used to check conformity with norms and pollutant concentrations fixed by legislation prior to embark on more detailed (and potentially expensive) analysis.

(iii) Continuous discharge monitoring can inform managers of the on-going functioning of their treatment plant. The detection of strong variation in effluents water quality can help identifying rapidly unstable processes. This early detection can help saving costs (avoiding damages and the payment of fines etc.). Water users might also use SMETs to obtain direct and continuous information on discharge quality and challenge the level of pollution tax they are paying. 
288 Overall, SMETs have four main advantages: (i) SMETs can help reducing the number 289 of spot samples and analyses; (ii) they can provide better information that reduces the 290 uncertainty of water quality measurements (for instance at risk or not to achieve good 291 status by 2015) and thus increases relevance of decisions taken to hamper 292 contamination; (iii) On site or in situ tools have the capability to deliver rapid results; 293 and, (iv), it ensures the quality of measurements for pollutants which concentration 294 degrades rapidly during shipping time.

\section{Constraints and opportunities throughout Europe}

296

297

298

299

300

301

302

303

304

305

Adapting monitoring strategies to comply with the WFD monitoring requirements needs to account for: 1) available (human) resources and budgets: 2) the preference given to existing monitoring and measurement techniques and to continuing past practices to avoid discontinuity in time series data. In this context, limited attention is given to innovations such as SMETs which advantages are rarely adequately considered. To the opposite, many limitations of SMETs are called for to justify that they are not really considered for WFD monitoring even if the technical report of the European Commission on the implementation of water monitoring requirements mentions their possible use [3]). These limitations can be summed up as follows:

\subsection{Regulatory constraints : SMETs lack normalisation}

Whereas the majority of analytical and sampling methods are normalized, this is not the case for SMETs. For many experts and countries, measurements and monitoring procedures require normalized methods. This is crucial for government agencies using the information to check compliance with regulation or to support court cases. In situ or on site analysis makes it difficult to have reproducibility or traceability, as water samples are not systematically taken back to lab for storage, although this could represent a viable option.

\subsection{Technical constraints}

For some SMETs, high limits of detection, low reliability (linked to regulatory constraint) and lack of in situ robustness are important factors that constraint today's application. Further, more technical information on the conditions of application of SMETs and on the interpretation of their results should be made available by those developing, distributing and selling these tools.

\subsection{Organisational constraints for users}

Increased use of SMETS might require organisational changes for departments in charge of monitoring and data analysis. Indeed the integration of SMETs in networks and surveys would imply adaptation of information systems, changes in task allocation and training needs. Continuous monitoring can in some cases require the building of complex databanks. The example of the Netherland Aqualarm system shows however the feasibility of such systems (see http://www.aqualarm.nl/). 
331 Laboratories and monitoring departments that decide to use SMETs will need to rely 332 on new partners that develop and sell these SMETs. Many SMETs are developed by 333 small companies or universities subsidiaries that might not have today the capacity or skills to market and disseminate their products. In some cases, necessary backup and assistance for their use might also not be sufficiently developed.

\subsection{Socio-cultural constraints : acceptance towards innovation}

Water quality and laboratory experts (mainly chemists) might not consider the shift from complex laboratory technologies to simple devices (a passive sampler is after all only a piece of plastic including a membrane...) as progress and innovation. Cultural habits might also lead to resistance from technicians that prefer to work in a secure laboratory instead of undertaking in-situ measurements which require additional field work.

\subsection{Economic constraints}

Public institutions recognise the limitations of their current monitoring networks. And they are keen to improve the effectiveness of monitoring system to deliver "better information” (i.e. information that better grasp the state of the aquatic environment). Often, however, limited financial resources and frozen budgets are mentioned as constraint to change. As a result, decisions that minimise change (and related potential hidden costs) are favoured. This applies to SMETs when considered in addition to the existing monitoring system and entailing higher costs. Assessments in the five European case studies have also highlighted the impact of labour costs (from a high $40 € /$ hour in Germany versus $4 € /$ hour in Latvia) on the overall cost of using SMETs. This turns SMETs to be more attractive from a financial \& budgetary point of view in low labour cost countries such as new European member states.

\subsection{Discussion: What to do for removing these barriers?}

As illustrated before, constraints to the wider use of SMETs are not limited to their technical characteristics. They also include cultural and economic constraints, in addition to classical reaction against innovation. Tackling existing constraints would require a proactive development strategy by those involved in their development and dissemination. To develop a standardisation protocol for the application of SMETs is seen as the cornerstone of this approach. Concentrating on a limited number of promising tools appears as essential to avoid potential end-users' confusion, to facilitate information and communication and to enhance trust. Training in the use of SMETs and in the interpretation of their results is also an essential component of this strategy.

Further work is also required to illustrate the potential impact of SMETs and their economic relevance. Indeed, the impact of better information on the state of the aquatic environment is often unknown and under-estimated. Integrating SMETs in existing monitoring networks will enhance the effectiveness of these networks in delivering adequate information. And better information can help targeting remediation measures and propose a more cost-effective way to protect water resources. 


\section{Conclusion}

377 The consultation of experts and practitioners carried out in five European countries confirms that SMETs are not perceived as substitute to standard analytical monitoring practices. Instead, their potential is as complementary tools delivering better information fast to achieve the objectives set by the WFD. While current large scale surveillance and operational networks offer some opportunities for the development of SMETs, their highest potential is in local surveys and investigations, e.g. (1) to assess the extent of pollution with emerging contaminants, or (2) to assess the extent and source of a groundwater pollution plume. The main strength of SMETs is clearly their ability to conduct quick on-site or in situ measurements, saving time and allowing the acquisition of a larger number of observations.

Extensive adoption of SMETs by water monitoring stakeholders is however not expected to take place in a very short time period. The deployment of SMETs is likely to generate additional costs resulting from training needs, adaptation of information systems, etc., that few organisations are willing to bear today. And staff reluctance to use methods providing results with high uncertainty that can not be compared to past data, that cannot be validated and that are not accredited, needs to be overcome. Private actors, to whom government agencies generally subcontract most of the sampling and analysis work, are also not likely to adopt innovative monitoring methods as they have often already invested time and money in high-tech instruments and corresponding human skills.

In the medium-term, the WFD management cycles and the need to upgrade monitoring systems will offer new windows of opportunity for integrating SMETs. It is expected that the larger number of practical applications, reduced costs (as more of them are used and produced) and efforts to develop and apply more systematically validation protocols and accreditation, will then provide the right conditions for SMETs to be given their due role in monitoring the state of the aquatic environment.

\section{Acknowledgments}

We acknowledge financial support of the European Union's Sixth Framework Programme (Contract SSPI-CT-2003- 502492; http://www.swift-wfd.com).

409 [1] European Commission. Official Journal of the European Communities L 327 410 (2000)

411 [2] European Commission. Official Journal of the European Commission L 348/84 412 (2008)

413 [3] L. Chery, C. Thouin, 6th international EWRA conference (European Water 414 Resources Association) (2005)

415 [4] M. Coquery, A. Morin, A. Bécue, B. Lepot, TrAC Trends in Analytical 416 Chemistry. 24 (2005) 2

417 [5] European Commission, WFD Technical report N.19 (2009) 
421 [7] R. Greenwood, G. A. Mills, B. Roig, TrAC Trends in Analytical Chemistry

422 Emerging tools as a new approach for water monitoring. 26 (2007) 4

423 [8] B. Roig, C. Valat, I. J. Allan, R. Greenwood, C. Berho, N. Guigues, G. A. Mills, 424 N. Ulitzur, TrAC Trends in Analytical Chemistry. 26 (2007) 4

425 [9] N. Guigues, C. Berho, S. Roy, J. C. Foucher, A. M. Fouillac, TrAC Trends in 426 Analytical Chemistry Emerging tools as a new approach for water monitoring. 26 427 (2007) 4

428 [10] H. Lückge, P. Strosser, N. Graveline, T. Dworak, J. D. Rinaudo, in: R. G.

429 Catherine Gonzalez Philippe Quevauviller (Eds.) Assessing the Impacts of Alternative 430 Monitoring Methods and Tools on Costs and Decision Making: Methodology and 431 Experience from case Studies, Rapid Chemical and Biological Techniques for Water 432 Monitoring, 2009, p.385. 